\title{
Dielectric Properties Extraction of Coplanar Propagation Waveguides on High Resistivity Silicon Substrates
}

\author{
Qing Lv \\ Hebei Normal University \\ Shi Jiazhuang, China \\ lvqing1017@163.com
}

Nicolas Defrance

Institute of Electronics, Microelectronics and

Nanotechnology, University Lille 1

Villeneuve d'Ascq Cedex - France

Corresponding author: nicolas.defrance @univ-lille1.fr

Kamel Haddadi

Institute of Electronics, Microelectronics and

Nanotechnology, University Lille 1

Villeneuve d'Ascq Cedex - France

Corresponding author: kamel.haddadi@univ-lille1.fr

\author{
Hua Zhao \\ Hebei Normal University \\ Shi Jiazhuang, China \\ zhaohualunwen@163.com
}

\author{
Chao Fu \\ Hebei Normal University \\ Shi Jiazhuang, China \\ fuchao1983@sina.com
}

\author{
LuJuan Ma \\ Hebei Normal University \\ Shi Jiazhuang, China \\ malujuan1985@126.com
}

\begin{abstract}
The dielectric properties of coplanar propagation waveguides (CPW) designed and fabricated on high resistivity silicon substrates are investigated. These CPWs exhibited transmission losses around $0.4 \mathrm{~dB} / \mathrm{mm}$ at $50 \mathrm{GHz}$. In particular, temperature-dependent measurements show very low deviations. The complex relative permittivity and loss angle tangents are extracted through adequate TEM analytical modeling. It is shown that the results are affected by parasitic peaks that are intrinsically linked with the extraction of the characteristic impedance. To overcome this issue, a numerical resolution is proposed, based on a modelling of the transmission parameter $S_{21}$ associated with the implementation of a bidimensionnal Newton-Raphson algorithm for the resolution of the inverse problem. Both methods give nearly identical results in terms of dielectric properties, confirming the validity and complementarity of the analytical and numerical models.
\end{abstract}

Keywords-High resistivity silicon (HR-Si); complex permittivity; coplanar waveguide; dielectric extraction; parameters $S$.

\section{INTRODUCTION}

The use of high-resistivity silicon (HR-Si) to address microwave and millimeter-wave applications has attracted the interest of the research community [1]. Thanks to its low-cost in comparison with III-V compound semiconductor substrate such as gallium arsenide ( $\mathrm{GaAs})$ or indium phosphide (InP), HR-Si is a suitable candidate for future monolithic and hybrid integrated circuits and systems. Furthermore, the wafer thermal conductivity and the wafer surface cristallinity are well understood. High quality coplanar waveguides (CPWs) on conventional $\mathrm{Si}$ or HR-Si substrates have been reported [2]-[10].

In this paper, we investigate the CPWs characteristics on HR-Si substrates for high power applications. In this kind of applications, the temperature can affect the devices and circuits performance. The CPWs are carefully designed and fabricated in order to achieve $50 \Omega$ impedance and low-loss up to $50 \mathrm{GHz}$. The broadband microwave measurements are performed to extract the insertion losses as a function of the temperature.

Additionally, from the measured data, the complex relative permittivity is extracted in the frequency range $100 \mathrm{MHz}-50 \mathrm{GHz}$.

\section{WAVEGUIDE FABRICATION}

Test structures consist of $10 \mathrm{~mm}$-length CPWs designed and fabricated on a $5 \mathrm{k} \Omega . \mathrm{cm}$ HR-Si substrate. The deposition process for the transmission lines was carried out by evaporation of gold in order to minimize the resistive losses in the metal at low frequencies. The associated metal thickness is $400 \mathrm{~nm}$. The microwave guide dimensions are defined to obtain $50 \Omega$ characteristic impedances (Fig .1) so that the loss characteristics can be directly extracted through $S$-parameters measurements. This results in line width $\mathrm{W}$ and slot width $\mathrm{S}$ of $54 \mu \mathrm{m}$ and $80 \mu \mathrm{m}$ respectively. The ground planes widths $\mathrm{GW}$ are set to $500 \mu \mathrm{m}$. 


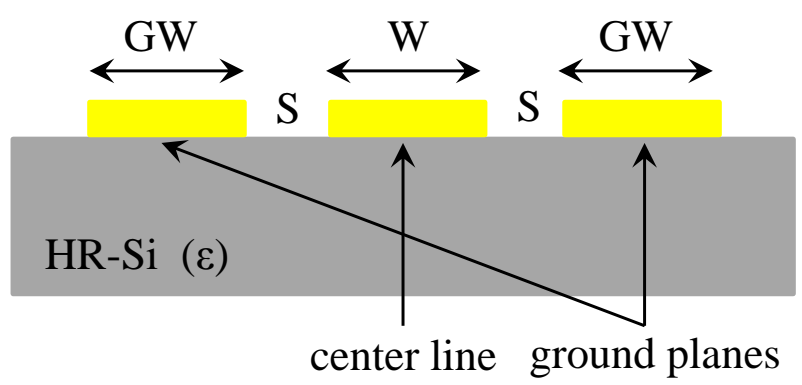

Figure 1. High-resistivity silicon coplanar propagation waveguide configuration.

\section{MEASUREMENT RESULTS}

Scattering parameters $S_{\mathrm{ij}}$ are measured using a vector network analyzer (VNA) and off-wafer line-reflect-match (LRMM) calibration over the $100 \mathrm{MHz}-50 \mathrm{GHz}$ frequency range. For temperature dependent measurements, a Karl Suss heating chuck is used with precision of $0.1 \mathrm{~K}$. From the observation of the reflection coefficient $S_{11}$ [Fig .2(a)], the transmission line is proved to be well matched. Indeed, it is noticed that the temperature has a negligible impact on the return loss on the frequency range considered. From the study of the transmission coefficient $S_{21}$ [Fig .2(b)], the variation on the insertion loss as a function of the temperature is also small. At $50 \mathrm{GHz}$, the normalized losses are around $0.4 \mathrm{~dB} / \mathrm{mm}$ whatever the temperature up to $100^{\circ} \mathrm{C}$.

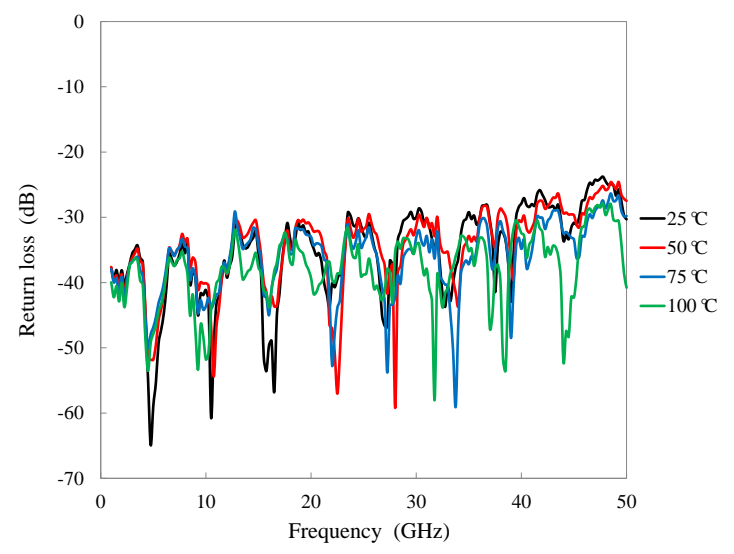

(a)

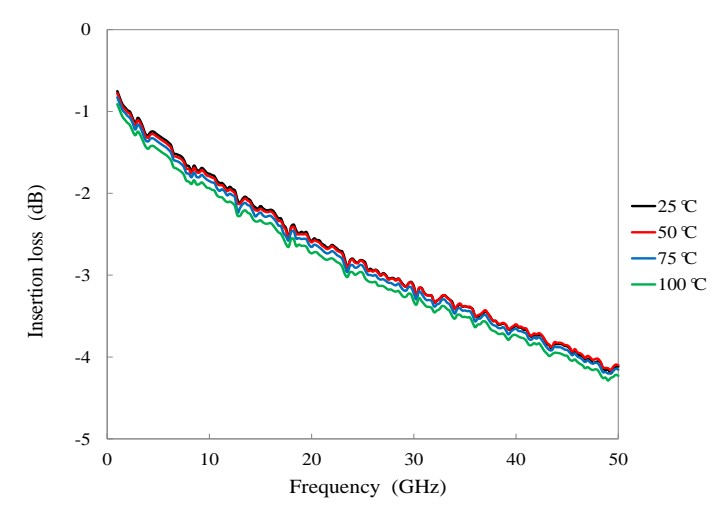

(b)

Figure 2. Measured $S$-parameters as a function of the chuck temperature. (a) Return loss. (b) Insertion loss.

\section{DIELECTRIC EXTRACTION}

Given the design of the processed transmission lines, the main propagation mode is assumed to be quasi-TEM. The relative complex permittivity of the HR-Si substrate is defined as $\varepsilon_{\mathrm{r}}=\varepsilon_{\mathrm{r}}{ }^{\prime}-\mathrm{j} \varepsilon_{\mathrm{r}}{ }^{\prime \prime}$ where $\varepsilon_{\mathrm{r}}{ }^{\prime}$ is the dielectric constant and $\varepsilon_{\mathrm{r}}{ }^{\prime \prime}$ is the loss factor. The propagation coefficient $\gamma$ and characteristic impedance $Z_{0}$ can be expressed as a function of the terms of the [ABCD] chain matrix (after a conversion of the measured $\left[S_{\mathrm{ij}}\right]$ parameters into[ABCD] parameters) by the following forms

$$
\begin{gathered}
\gamma=\frac{\ln \left(\mathrm{A}+\sqrt{\mathrm{A}^{2}-1}\right)}{1} \\
\mathrm{Z}_{0}=\sqrt{\frac{\mathrm{B}}{\mathrm{C}}}
\end{gathered}
$$

Where the parameter $l$ represents the physical length of the coplanar line. From these two quantities, the whole four lumped parameters (R,L,C,G) model can be extracted, assuming the transmission line can be represented as a two-wire line (Fig .3). The $\mathrm{R}$ parameter relates the resistivity of the deposited gold line.

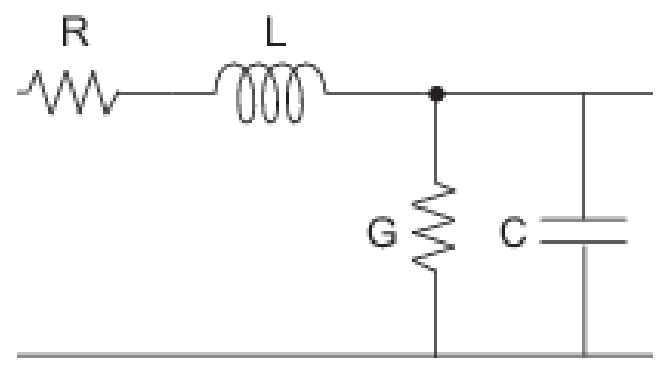

Figure 3. Distributed equivalent-circuit model of the CPW. The elements R, L, G and C are defined per length.

In particular, the knowledge of $\mathrm{C}$ and $\mathrm{G}$ (the parallel elements) permit to determine the complex dielectric constant of the substrate, hence the loss angle $\operatorname{tangent} \tan \delta$. The involved relationships rely on the quasi-TEM model from Heinrich and can be written as [11]

$$
\begin{aligned}
& \mathrm{C}=2 \varepsilon_{0} \times\left(\mathrm{F}_{\text {up }}+\varepsilon_{\mathrm{r}} \times \mathrm{F}_{\text {low }}\right) \\
& \mathrm{G}=2 \omega \varepsilon_{0} \varepsilon_{\mathrm{r}} \times \tan (\delta) \mathrm{F}_{\text {low }} .
\end{aligned}
$$

Where $F_{\text {up }}$ and $F_{\text {low }}$ are geometrical based weight functions [11], $\omega$ is the angular pulsation and tan $(\delta)$ is the loss angle tangent of dielectric. The model validity can be assessed by the observation of the $\beta / \beta 0$ behavior (not shown here) where $\beta\left(=2 \pi / \lambda_{\mathrm{g}}\right)$ and $\beta_{0}\left(=2 \pi / \lambda_{0}\right)$ are the phase constants along the CPW and in vacuum, respectively. 
From the measured $S$-parameters and the geometrical parameters of the CPW, the RLCG extraction algorithm has been implemented. Fig .4 presents the frequency behavior of the reactive elements $\mathrm{L}$ and $\mathrm{C}$.

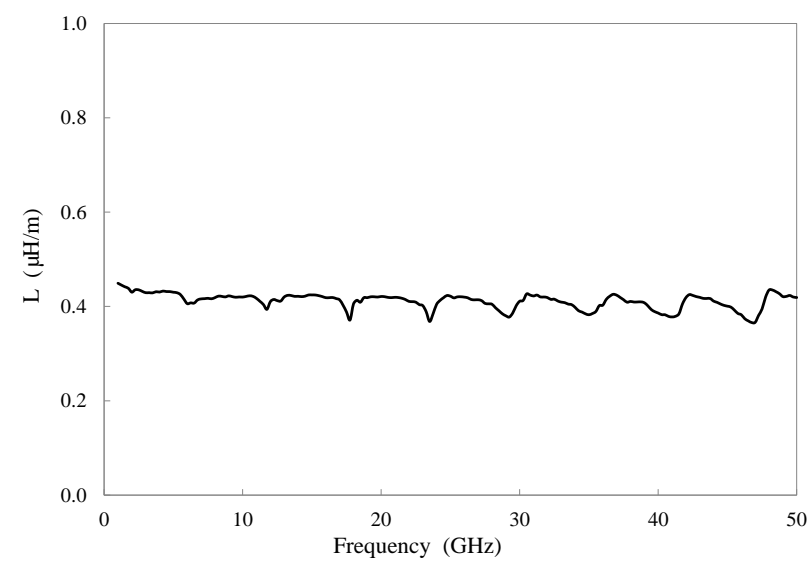

(a)

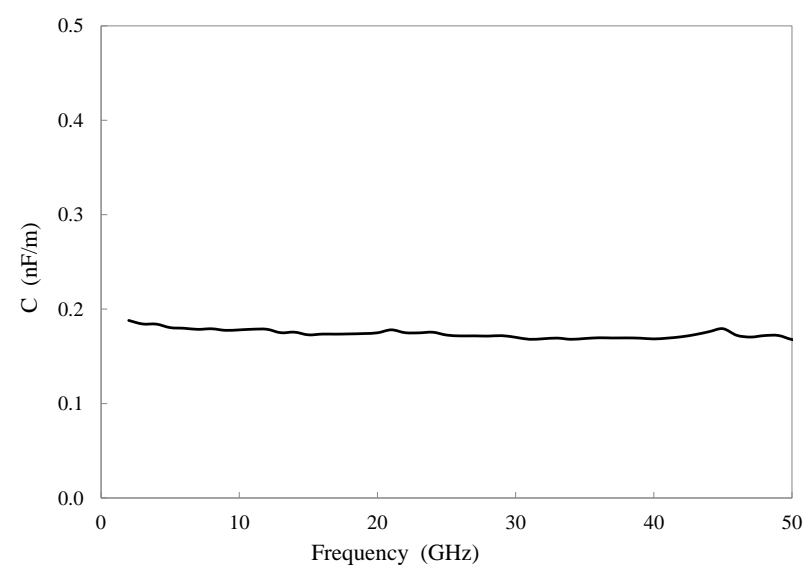

(b)

Figure 4. Extracted CPW line (a) inductance $\mathrm{L}$ and (b) capacitance $\mathrm{C}$ as a function of the frequency.

From these data, both measured responses are relatively flat demonstrating the accuracy of the model. Resonance peaks that appear in Fig .4(a) are mainly attributed to the extraction algorithm. On can mention that such undesirable peaks can be easily eliminated by using measurements considering smaller CPW lengths but this can induce parasitic cross-over capacitance effects that can affect the accuracy of the results. Another solution is to compare the dielectric extraction from data related to different transmission lengths.

The relative complex permittivity of the substrate can be calculated from $\mathrm{C}$ and $\mathrm{G}$ [11] using conformal mapping formula based on elliptic functions. Fig .5 shows the obtained data. The results obtained from the model give permittivity extracted around 12 for the HR-Si substrate. From the knowledge of the imaginary quantity $\varepsilon_{\mathrm{r}}$ ", we can deduced the frequency dependency of the loss angle tangent corresponding to the ratio $\varepsilon_{\mathrm{r}}$ "' $/ \varepsilon_{\mathrm{r}}$ ' [Fig .5(b)]. From the data, the median loss tangent is estimated between 0.1 and 0.2 and does not show major deviation when the temperature increases. The peaks are attributed to the extraction algorithm and are not of physical origin, hence a new extraction strategy need to be embedded. This is considered in the next paragraph.To address the issue coming from the first order quasi-TEM analytical model, a numerical resolution based on Newton-Raphson algorithm can be considered. In this context, the propagation coefficient $\gamma$ can then be written as $\gamma=\alpha+j \beta$ with

$$
\begin{aligned}
& \alpha=\frac{2 \pi}{\lambda_{0}} \sqrt{\frac{1}{2}\left(|\varepsilon|-\varepsilon^{\prime}\right)} \\
& \beta=\frac{2 \pi}{\lambda_{0}} \sqrt{\frac{1}{2}\left(|\varepsilon|+\varepsilon^{\prime}\right)} .
\end{aligned}
$$

where $\alpha$ represents the lossy behaviour of the media and $\beta$ the propagation phase shift. The modelling method is based on the minimization of a cost function considering the measured transmission parameter

$$
S_{21 . \text { model }}-S_{21 . \text { measured }}=0 \text {. }
$$

A Newton-Raphson bi-dimensional algorithm is used to extract the values of $\varepsilon^{\prime}$ and $\varepsilon^{\prime \prime}$. The results are presented on Fig .5. It is shown that this first order modelling gives similar results than those extracted through quasi-TEM Heinrich model. Additional analysis was carried out extracting the loss angle tangent as function of the chuck temperature; the results are given in the inset of Fig .5b at the test frequency $50 \mathrm{GHz}$. We show that our assumptions are confirmed since the relative variation of the $\tan (\delta)$ is less than $1 \%$ over the $25^{\circ} \mathrm{C}-100^{\circ} \mathrm{C}$ temperature range.

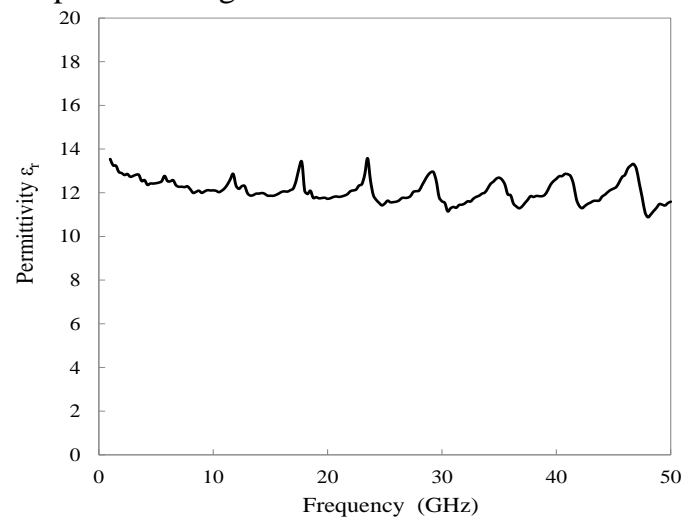

(a)

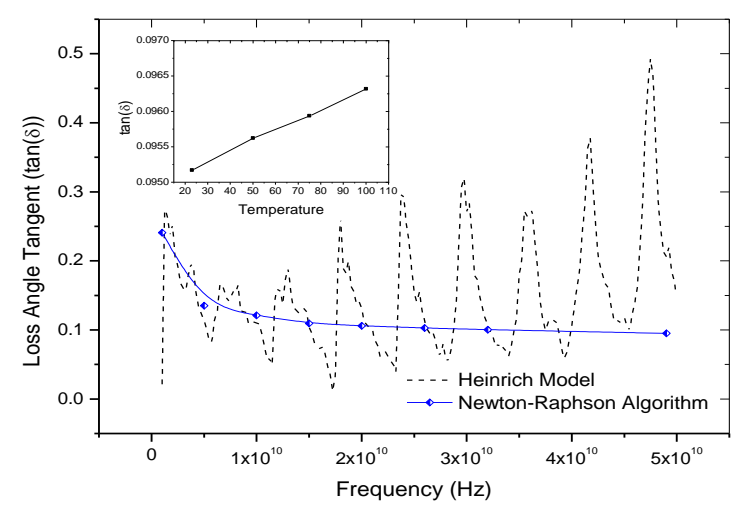

(b)

Figure 5. Extracted complex permititivty of the HR-Si CPW as a function of the frequency. (a) Permitivity. (b) Loss angle tangent. 


\section{CONCLUSION}

High-resistivity silicon technology for microwave and millimeter-wave transmission in coplanar waveguides has been investigated as a function of the temperature. It has been shown that the heating between $25^{\circ} \mathrm{C}$ and $100^{\circ} \mathrm{C}$ has a negligible impact on the measured RF performances. From the $S$-parameters, the complex permittivity is extracted in the range $100 \mathrm{MHz}-50 \mathrm{GHz}$. Future works will include the study of miniaturized coplanar waveguides and formulation of dedicated modeling to address next generation of micro- and nanoelectronic devices.

\section{ACKNOWLEDGEMENTS}

This work was financially supported by the Hebei Province Education Office Foundation (QN2014116), Hebei Province Education Office Foundation (QN2014071), Hebei Normal University Youth Foundation (L2010Q10), and Hebei Normal University Doctoral Foundation (L2012B15).

\section{REFERENCES}

[1] P. J. Stabile, A. Rosen, W. M. Janton, A. Gombar, M. Kolan, "Millimeter wave silicon device and integrated circuit technology", MTT-S Dig., vol. 84, pp.448-450.

[2] J. Büchler (Buchler), E. Kasper, P. Russer, K. M. Strohm, "Silicon high-resistivity substrate millimeter wave technology", IEEE Trans Microwave Theory Tech., vol. MTT-34, pp.1516-1521, Dec. 1986.

[3] M. H. Hanes, A. K. Agarwal, T. W. O'Keefe, H. M. Hobgood, J. R. Szedon, T. J. Smith, R. R. Siergiej, P. G. McMullin, H. C. Nathanson, M. C. Driver, R. N. Thomas, "MICROX-an all- silicon technology for monolithic microwave integrated circuits", IEEE Electron Device Lett., vol. 14, pp.219-221, May 1993.

[4] A. C. Reyes, S. M. El-Ghazaly, S. Dorn, M. Dydyk, D. K Schroder, H. Patterson, "High-resistivity silicon as a microwave substrate", Proc. Electronic Components and Technology Conf., pp.382-391.

[5] B. Rejaei, K. T. Ng, C. Floerkemeier, N. P. Pham, L. K. Nanver, J. N. Burghartz, "Integrated transmission lines on high-resistivity silicon: coplanar waveguides or microstrips?", Proc. ESSDERC pp.460-463

[6] C. Schöllhorn (Schollhorn), W. Zhao, M. Morschbach, E. Kasper, "Attenuation mechanism of aluminum millimeter wave coplanar waveguides on silicon", IEEE Trans. Electron Devices, vol. 50, pp.740-746, March 2000.

[7] H. S. Gamble, B. M. Armstrong, S. J. N. Mitchell, Y. Wu, V. F. Fusco, J. A. C. Stewart, "Low-loss CPW lines on surface stabilized high-resistivity silicon", IEEE Microwave Guided Wave Lett. vol. 9, pp.395-397, Oct. 1999.

[8] E. Valetta, J. van Beek, A. den Dekke, N. Pulsford, H. F. F. Jos, L. C. N. de Vreede, L. K. Nanver, J. N. Burghartz, "Design and characterization of integrated passive elements on high-ohmic silicon", MTT-S Dig., vol. 2, pp.1235-1238.

[9] A. B. M. Jansman, J. T. M. van Beek, M. H. W. M. Van Delden, A L. A. M. Kemmeren, A. D. Dekker, F. P. Widdershoven, "Elimination of accumulation charge effects for high-resistivity silicon substrate", Proc. ESSDERC, pp.3-6.

[10] K. Benaissa, Y. Jau-Yuann, D. Crenshaw, B. Williams, S. Sridhar, J. Ai, G. Boselli, Z. Song, T. Shaoping, S. Ashburn, P. Madhani, T. Blythe, N. Mahalingam, H. S. Shichijo, "RF-CMOS on highresistivity substrates for system-on-chip applications", IEEE Trans. Electron Devices, vol. 50, pp.567-576, March 2003.

[11] Wolfgang Heinrich, "Quai-TEM Description of MMIC Coplanar Lines including Conductor-Lass Effects”, IEEE Trans. Microwave Theory Tech., vol. 41, no. 1, pp. 45-52, Jan. 1993. 